\title{
Impacto de la viremia de bajo grado en el riesgo de fallo virológico en pacientes con infección por VIH-1
}

\author{
Impact of low-grade viremia on the risk of virological failure in patients with HIV-1 infection \\ on antiretroviral therapy
}

Romina Viceconte1, Verónica Cisneros', Diego Sánchez Thomas', Florencia Spacapan?',

María L. Fernández Ventura', Carla Petriglieri' y Gustavo Lopardo'

${ }^{1}$ Fundación Centro de Estudios Infectológicos (FUNCEI). Buenos Aires, Argentina.

Conflictos de interés: Gustavo Lopardo: advisor BMS, Gilead. Disertante: BMS, Gilead. El resto de los autores no posee conflictos de interés.

Fuente de financiamiento: Ninguna.

Recibido (segunda versión): 13 de agosto de 2020 / Aceptado: 17 de agosto de 2020

\section{Resumen}

Introducción: Cargas virales (CV) entre 20-200 copias/mL se consideran cargas virales de bajo grado (CVBG). Su implicancia clínica y manejo no han sido definidos. Objetivo: Evaluar el impacto de CVBG en el riesgo de desarrollo posterior de fallo virológico (FV). Pacientes $y$ Métodos: Se incluyeron pacientes $\geq 18$ años, desde enero de 2009 a diciembre de 2019, con infección por $\mathrm{VIH}-1$ con $\mathrm{CV}<20$ copias $/ \mathrm{mL}$, por un mínimo de seis meses y/o en dos muestras consecutivas bajo tratamiento anti-retroviral. Se realizó seguimiento de las CV estratificándolas: $\mathrm{CV}<20$ copias/mL, CVBG (20-50 copias/mL y 51-200 copias/mL) y FV. Mediana de seguimiento 25 meses (IQR 15-31). Resultados: Fueron incluidos 1.416 pacientes con $\mathrm{CV}<20$ copias/ $\mathrm{mL}$ bajo TARV. De ellos, 797 permanecieron con $\mathrm{CV}<20$ copias $/ \mathrm{mL}$ durante el seguimiento, 144 presentaron $\mathrm{CV}$ entre $20-50$ copias $/ \mathrm{mL}$, 384 entre 51-200 copias/mL y 91 presentaron FV sin CVBG previa. De los 528 pacientes que tuvieron CVBG, $110(20,1 \%)$ fallaron, riesgo 3,45 veces superior respecto a los que no tuvieron CVBG previa. El riesgo de $\mathrm{FV}$ fue 3,27 mayor para aquellos que tuvieron $\mathrm{CVBG}$ entre 51-200 copias/mL vs 20-50 copias/mL. Discusión: El estudio permite relacionar la CVBG con el FV posterior, siendo el mayor riesgo CVBG entre 51-200 copias $/ \mathrm{mL}$.

Palabras clave: VIH; carga viral; fallo virológico; viremia de bajo grado.

\begin{abstract}
Background: Viral loads (VL) between 20-200 copies/mL are considered low-grade viral loads (LGVL). Its clinical implications and management have not been defined. Aim: To evaluate the impact of LGVL on the risk of subsequent development of virological failure (VF). Methods: Patients $\geq 18$ years, with HIV-1 infection who had VL $<20$ copies/mL for at least six months and/or in two consecutive samples under antiretroviral therapy (ART) were included, between January $1^{\text {st }}, 2009$ and December 31, 2019. Follow-up of the VLs was carried out stratifying them in VL $<20$ copies/mL, LGVL (20-50 copies/mL and 51-200 copies/mL) and VF. Median follow-up 25 months (IQR 15-31). Results: 1,416 patients were included who reached $\mathrm{VL}<20$ copies/ml under ART, 797 patients remained with $\mathrm{CV}<20$ copies $/ \mathrm{mL}$ during follow-up, 144 patients had VL between 21-50 copies/mL, 384 between 51-200 copies/mL and 91 had VF without previous LGVL. Out of 528 patients who had LGVL, 110 failed, risk 3.45 times higher than those who had no previous LGVL. Risk 3.27 times higher of VF for those who had LGVL between 51-200 copies/mL compared to 20-50 copies/mL. Discussion: The study allows to relate the LGVL with VF. This association was observed more frequently with LGVL between 51-200 copies $/ \mathrm{mL}$

Keywords: HIV; viral load; virological failure; low-level viremia.
\end{abstract}


Como conclusión, refieren que la CVBG es frecuente, y que menos de $5 \%$ de los pacientes que experimentan CVBG, evolucionan a FV dentro del año de seguimiento, sin documentar resistencia viral. Los autores consideraron que los factores que contribuyeron a la presencia de CVBG intermitente o permanente podrían ser la existencia de reservorios virales, variaciones o error de laboratorio, continua replicación viral en tejidos y disminución de la adherencia. La CVBG por períodos prolongados podría estar asociada a activación inmune, aunque no se puede predecir el impacto clínico que conlleva ${ }^{5}$.

Teira y cols., evaluaron 21.480 pacientes que habían iniciado TARV. De ellos, 824 pacientes tuvieron CVBG definida como CV entre 20 y 50 copias/mL luego de la supresión viral, no encontrando asociación entre el bajo nivel de replicación y el desarrollo de FV. Cuando evaluaron al grupo con CVBG entre 50 y 200 copias $/ \mathrm{mL}$, encontraron una mayor tendencia de evolucionar a $\mathrm{FV}^{6}$.

Hermans y cols., publicaron un estudio observacional, multicéntrico, realizado en 57 clínicas de Sudáfrica, desde 2007 a 2016, en el que evaluaron 70.930 pacientes. Definieron la CVBG a la CV ocurrida al menos una vez entre 51-999 copias/mL. Definieron como FV a una o más CV mayores a 1.000 copias/mL. La CVBG ocurrió en 23\% (16.013) de los pacientes, con una incidencia de 11,5 por 100 personas/año de seguimiento. El FV se produjo en $22 \%$ de los pacientes (14.380). La CVBG estuvo asociada con el aumento de FV comparado con los pacientes que mantuvieron la CV suprimida. Sus resultados demostraron que a mayor valor de CVBG, mayor riesgo de presentar FV posterior. Los autores concluyeron que CVBG ocurre frecuentemente, aumentando el riesgo de FV con el consiguiente cambio a segunda línea de TARV ${ }^{7}$.

En un reciente estudio publicado Joya y cols., evaluaron la asociación entre la persistencia de CVBG (pCVBG) y el FV. Se utilizaron datos retrospectivos de US Military HIV Natural History Study (NHS), donde incluyeron 2.006 sujetos que habían iniciado TARV luego del 1 de enero de 1996 y presentaban al menos dos CV menores a 50 copias $/ \mathrm{mL}$, a los seis meses de haber comenzado el tratamiento. El seguimiento finalizó en octubre de 2017. Dividieron a los pacientes en cuatro categorías: pacientes con $\mathrm{CV}$ menos de 50 copias/mL, pacientes con CV de alto grado, entre 200 y 1.000 copias/mL no consecutivas y otros con CVBG entre 50 y 199 copias/mL, que a su vez se subdividían en CVBG intermitentes (si la CVBG aparecía en menos de $25 \%$ de los resultados) o CVBG persistentes (si era mayor de $25 \%$ ). Los resultados mostraron que la CVBG persistente y las viremias de alto grado se asociaron a mayor riesgo de $\mathrm{FV}^{8}$.

No existe consenso en relación con el manejo de pacientes con CVBG dado que el riesgo de resistencia es bajo. Se sugiere controlar la CV cada tres meses, para 
evaluar la necesidad de cambios en el TARV (recomendación AIII) ${ }^{3}$.

El objetivo del presente estudio es analizar el riesgo de FV en sujetos que habiendo alcanzado la indetectabilidad presentaron CVBG.

\section{Objetivos}

Primario:

- Evaluar el impacto de CVBG en el posterior riesgo de $\mathrm{FV}$, analizando el riesgo que existe con CVBG entre 20 - y 50 copias $/ \mathrm{mL}$ y entre 51 y 200 copias $/ \mathrm{mL}$.

Secundario:

- Analizar la posible relación entre el régimen de TARV que recibía el paciente en el momento del desarrollo de CVBG conteniendo inhibidores de la proteasa (IP) o inhibidores no nucleosídicos de la transcriptasa inversa (INNTI) con el FV posterior.

\section{Materiales y Métodos}

Estudio observacional, longitudinal, retrospectivo. Se revisaron las historias clínicas de pacientes asistidos entre enero de 2009 y diciembre de 2019 en el Centro de Estudios Infectológicos, sucursal Barrio Norte, de la Ciudad Autónoma de Buenos Aires, Argentina.

El estudio fue conducido de acuerdo con los principios éticos que tienen su origen en la Declaración de Helsinki y con las normas regulatorias correspondientes. Se realizó bajo la ley 25.326 de protección de los datos personales.

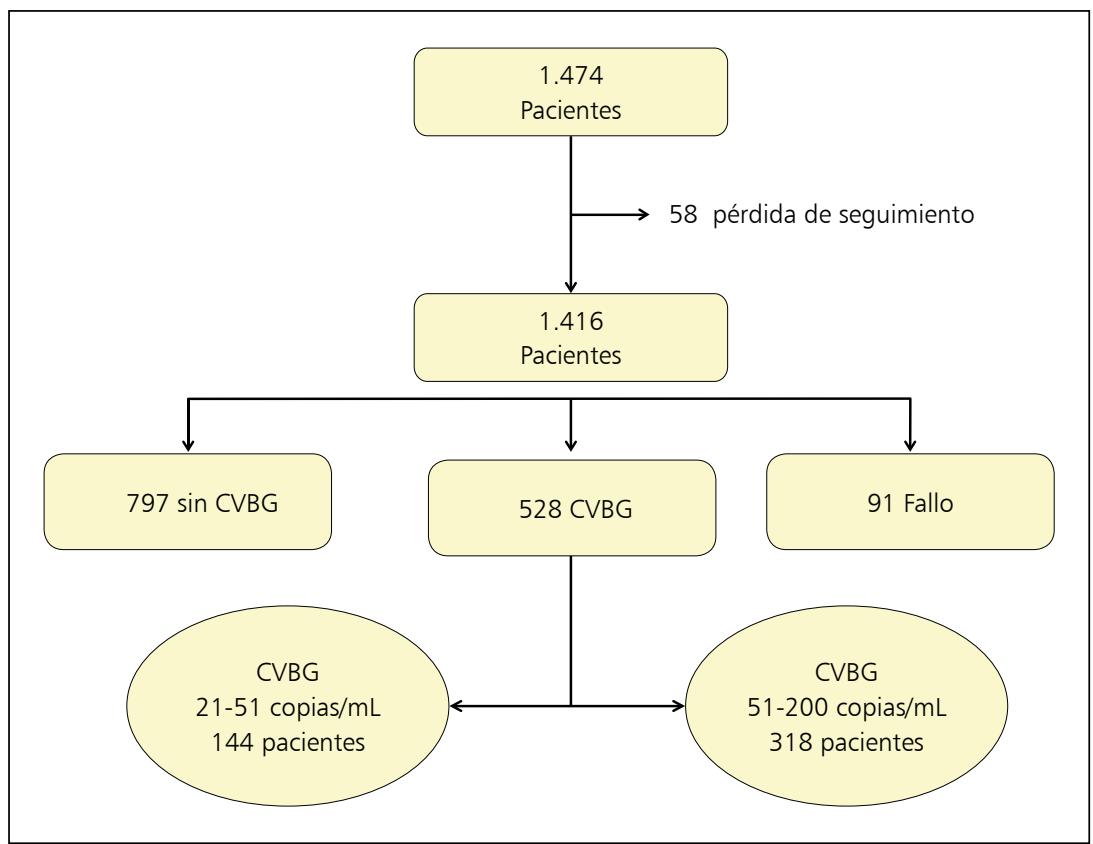

Figura 1. Estratificación de la población estudiada según los valores de CV.
Se mantuvo la confidencialidad de los datos, asegurando su protección integral.

Fueron incluidos los pacientes $\geq 18$ años con infección documentada por VIH-1 con $\mathrm{CV}<20$ copias $/ \mathrm{mL}$ en dos muestras consecutivas separadas por un mínimo de seis meses bajo TARV.

Se realizó el seguimiento de las $\mathrm{CV}$ de estos pacientes durante el período estudiado y se los clasificó en tres grupos:

Grupo 1: pacientes con CV suprimida, persistentemente $<20$ copias $/ \mathrm{mL}$.

Grupo 2: pacientes que presentaban CVBG: entre 20 y 50 copias $/ \mathrm{mL}$ y entre 51 y 200 copias $/ \mathrm{mL}$.

Grupo 3: pacientes con CV $>200$ copias/mL confirmada sin CVBG previa (FV).

Dentro de la base de datos se incluyó sexo, edad, tiempo de infección, valor de linfocitos T (LT) CD4 al momento del diagnóstico, tiempo transcurrido a la aparición de la CVBG o de $\mathrm{FV}$, valor de la $\mathrm{CV}$ en el momento de la CVBG y TARV que recibía el paciente en ese momento. Todos los pacientes tuvieron al menos 12 meses de seguimiento y la mediana de seguimiento fue 25 meses (RIQ 15-31).

Para el análisis estadístico se utilizó estadística descriptiva y test de $\chi^{2}$. Se usaron curvas de sobrevida para estimar el tiempo al evento y análisis de Kaplan- Meier. La asociación entre las características basales de los pacientes y la durabilidad del TARV se estimó utilizando análisis univariado y multivariado y modelo de riesgo proporcional (Cox). Se utilizaron los programas estadísticos SPSS (SPSS versión 15.0) y STATA (versión 8.0).

\section{Resultados}

De un total de 1.474 pacientes evaluados, ingresaron al estudio 1.416. Se perdió el seguimiento de 58 pacientes que fueron derivados a otros centros (Figura 1). El 87,4\% de los sujetos eran de sexo masculino, presentando una edad media de 45 años (R 20-84) (Tabla 1).

El valor de la mediana de LT CD4 fue de 551 céls $/ \mathrm{mm}^{3}$ (RIQ 402-719) al momento de la inclusión en el estudio y el tiempo medio de infección fue de 63,5 meses (R 1-324). La mediana de seguimiento fue de 25 meses (RIQ 15-31).

De los 1.416 pacientes evaluables, 797 (56,3\%) correspondieron al grupo 1 (CV indetectable en forma persistente), 528 (37,3\%) al grupo 2, de los cuales 144 con CVBG entre 20 y 50 copias/mL y 384 con CV entre 51 y 200 copias/mL, y $91(6,4 \%)$ al grupo 3 (FV).

De los 528 pacientes del grupo 2; $110(20,1 \%)$ desarrollaron FV. El riesgo de FV fue de 3.45 veces mayor de evolucionar a FV en este grupo, en comparación con los pacientes que permanecieron con $\mathrm{CV}$ indetectable. Odds ratio 3,45 (IC 95\% 2,59-4,77) $(\mathrm{p}<0,01)$. 
Se analizaron los pacientes que presentaban CVBG entre 21 y 50 copias/mL (n: 144) y la evolución al FV se observó en seis pacientes $(4,16 \%)$. El riesgo de FV fue de 1,48 veces mayor de evolucionar a FV en este grupo que en quienes mantienen la $\mathrm{CV}$ indetectable. Odds ratio 1,48 (IC 95\% 1,37-1,89) $(\mathrm{p}<0,01)$.

Entre los pacientes que presentaron CVBG entre 51 y 200 copias/mL $(n=384)$ la evolución a FV se observó en 104 pacientes (27\%). El riesgo de FV fue 2,75 veces mayor de evolucionar al FV en este grupo, en comparación con los pacientes que permanecieron indetectables. Odds ratio 2,75 (IC 95\%. 1,45-6,84) ( $\mathrm{p}<0,01)$.

También se compararon ambos grupos: pacientes que presentaban CVBG 21 a 50 copias/mL y CVBG 51 a 200 copias/mL y el riesgo de evolución a FV. Se obtuvo un riesgo de 3,27 veces mayor de presentar FV en el grupo con CV 51 a 200 copias/mL en relación a los pacientes que presentaron CVBG 21 a 50 copias $/ \mathrm{mL}$, Odds ratio $3,27$ (IC 95\% 2,05-5,22) ( $\mathrm{p}<0,01)$.

El objetivo secundario del presente estudio fue analizar el tipo de régimen ARV que recibían los pacientes en el momento de presentar CVBG. Los regímenes de TARV consistentes en 2 INTI más 1 INNTI o 1 IP representaron $87 \%$ del total de los esquemas de TARV que recibía la población estudiada. El análisis de los 528 sujetos que presentaron CVBG mostró que de los pacientes que fueron tratados con regímenes que incluían dos INTI más un IP $17 \%$ presentaron $\mathrm{FV}$ y $7 \%$ de los pacientes tratados con dos INTI más un INNTI. Odds ratio 2,58 (IC 1,88-3,52) $(\mathrm{p}<0,01)$.

\section{Discusión}

Los resultados del presente estudio sugieren que los pacientes infectados con VIH que se encuentran recibiendo TARV y que lograron alcanzar $\mathrm{CV}$ indetectable, si presentan CVBG definida como valores de CV entre 21 y 200 copias/m, tienen un riesgo incrementado de evolucionar a $\mathrm{FV}$ en relación con los pacientes que mantienen la $\mathrm{CV}<20$ copias/mL, y que, a mayor $\mathrm{CVBG}$, es mayor el riesgo de presentar FV.

Los estudios publicados hasta el presente han demostrado hallazgos contradictorios en relación con la presencia de CVBG y el riesgo posterior de FV. Algunas publicaciones han observado que la presencia de CVBG es frecuente y que no se asocia con el desarrollo posterior de $\mathrm{FV}^{5,9}$. Otros estudios mostraron que los pacientes con CVBG tuvieron mayor riesgo de evolucionar a FV que los pacientes que se mantuvieron persistentemente indetectables ${ }^{4,6,7}$. Dos estudios recientemente publicados sugieren que la persistencia de CVBG es la que presenta más riesgo de $\mathrm{FV}$, no así la $\mathrm{CVBG}$ aislada ${ }^{8,10}$.

En nuestro estudio se observó el desarrollo CVBG en

\begin{tabular}{|c|c|c|}
\hline & $\mathbf{n}$ & (\%) \\
\hline \multicolumn{3}{|l|}{ Sexo } \\
\hline Masculino & 1.246 & (88) \\
\hline Femenino & 170 & (12) \\
\hline \multicolumn{3}{|l|}{ Edad (años) } \\
\hline Menos de 24 & 5 & $(0,3)$ \\
\hline $24-39$ & 421 & $(29,7)$ \\
\hline $40-49$ & 529 & $(37,3)$ \\
\hline Mayor 50 & 461 & $(32,5)$ \\
\hline \multicolumn{3}{|c|}{ Recuento de LT CD4 (céls/mm³) } \\
\hline Menor 200 & 79 & $(5,5)$ \\
\hline $200-499$ & 497 & $(35,1)$ \\
\hline Mayor a 500 & 828 & $(58,4)$ \\
\hline$S / D$ & 12 & $(0,8)$ \\
\hline \multicolumn{3}{|l|}{ TARV } \\
\hline INNTI & 888 & $(62,7)$ \\
\hline IP & 492 & $(34,7)$ \\
\hline$\|$ & 10 & $(0,7)$ \\
\hline Otros & 25 & $(1,7)$ \\
\hline
\end{tabular}

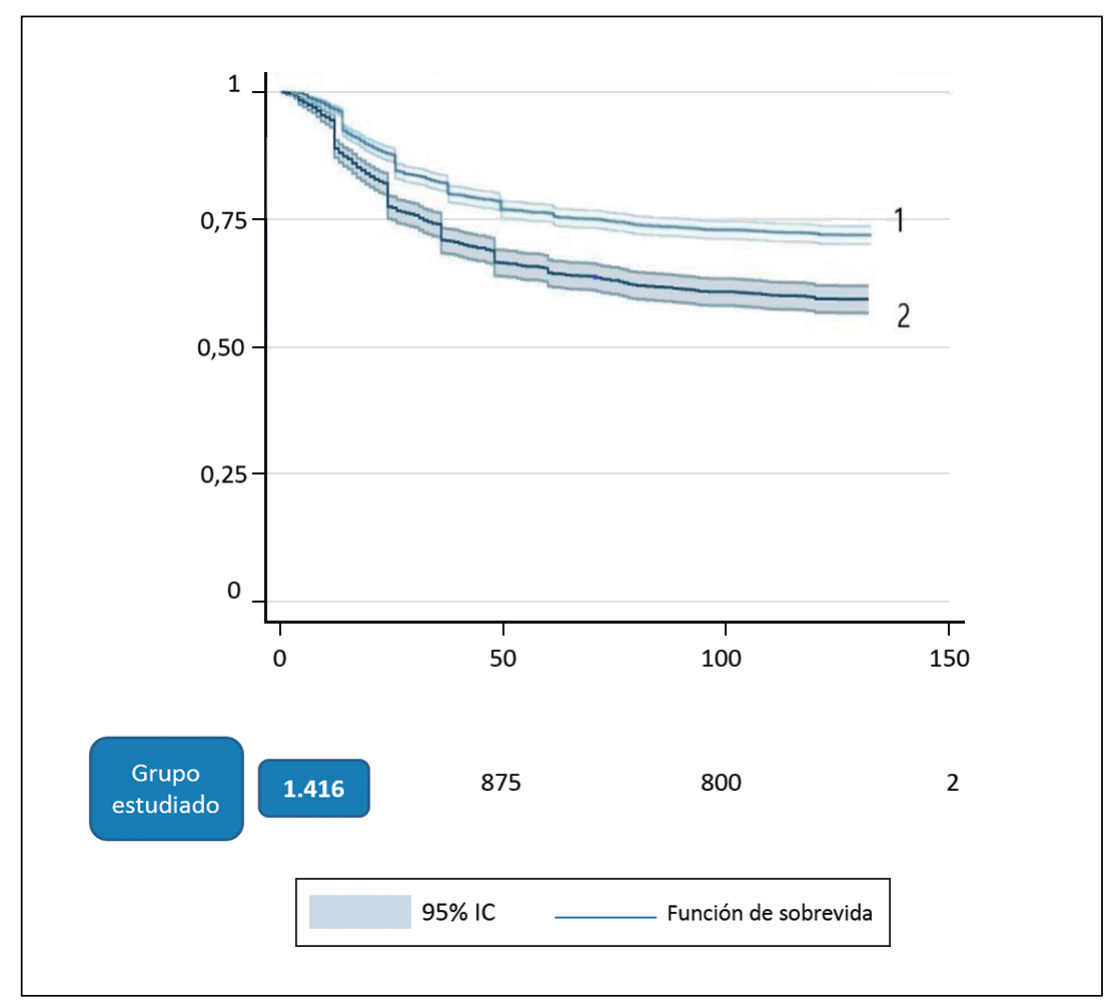

Figura 2. Curva de Kaplan-Meier. Curva 1 CV 21-50 copias/mL. Curva 2: CV 51-200 copias/mL. 
$37,3 \%$ de los pacientes evaluados, similar a otros estudios realizados, donde se evidenció un rango de 20 a $40 \%$ de $\mathrm{CVBG}^{11}$. Los resultados del presente estudio evidencian el mayor riesgo de evolucionar a $\mathrm{FV}$ en comparación con los pacientes que mantienen $\mathrm{CV}$ persistentemente suprimida, presentando mayor riesgo los pacientes que se encuentran en el estrato de 51 a 200 copias $/ \mathrm{mL}$ en comparación con el grupo con $\mathrm{CV}$ de 20 a 50 copias $/ \mathrm{mL}$.

La fuente de la CVBG es controversial y no ha sido establecida con certeza. Podría ser explicada por liberación viral secundaria a la activación de LT CD4 latentes infectados. Otra posibilidad es la replicación del virus como consecuencia de una terapia sub-óptima, especialmente dentro de los reservorios anatómicos menos accesibles al TARV, facilitados por las variaciones en las concentraciones de los ARV, que pueden ser atribuidas por cuestiones farmacocinéticas. La incompleta adherencia al TARV y/o a la emergencia de resistencia ARV asociada a mutaciones son otras posibles causas de $\mathrm{CVBG}^{5}$.

Algunos estudios sugieren que la presencia de CVBG continua, por períodos prolongados y cuando la replicación es de mayor valor, podría favorecer el desarrollo de FV más frecuentemente que un registro de CVBG aislado, y que este resultado podría ser por diferentes factores como la mala adherencia del paciente o las variaciones entre los métodos de cuantificación de la CV. Este escenario podría aumentar el riesgo potencial de desarrollar mutaciones ${ }^{5}$.

El objetivo secundario de nuestro estudio fue analizar la asociación entre el régimen de TARV que el paciente recibía en el momento del desarrollo de CVBG, y el posterior desarrollo de FV. El análisis mostró que hubo un mayor riesgo de desarrollar FV en aquellos que recibían regímenes ARV incluyendo IP en comparación con INNTI. Una hipótesis que podría justificar la tendencia al mayor FV de los esquemas que incluyen IP, es la toxicidad asociada a esta familia de fármacos y que ésta genere dificultades de adherencia.

Limitaciones del presente estudio son el diseño observacional y retrospectivo, y el tamaño reducido de la muestra. El diseño retrospectivo no permite analizar la adherencia de los pacientes y el impacto que ésta pudiera tener en el desarrollo de CVBG y/o en el posterior FV.

En conclusión, el presente estudio provee información de una población de pacientes asistidos en un centro de la ciudad de Buenos Aires que permite entender mejor el impacto de la presencia de CVBG, dando como resultado que la presencia de CVBG presenta mayor riesgo de evolucionar a FV, y que a mayor CVBG, también mayor es el riesgo de $\mathrm{FV}$.

Agradecimientos: Los autores agradecen la colaboración de los participantes, sin ellos, este trabajo no hubiera sido posible. Además, agradecemos el apoyo del personal que colaboró en el desarrollo del estudio, a quienes mencionamos a continuación: Luchetti P, Berardo T, Tortoriello A, Glasman P, Altamirano M, Masid E, Fiori A.

\section{Referencias bibliográficas}

1.- VII Consenso Argentino de terapia antirretroviral 2018-2019. Mayo 2019. SADI. https://www.sadi.org.ar/guiasrecomendaciones-y-consensos (acceso el 31 de julio de 2020).

2.- Documento de consenso de GeSIDA/plan nacional sobre el SIDA respecto al tratamiento antirretroviral en adultos infectados por el virus de la inmunodeficiencia humana. Actualización julio 2020. http://gesida-seimc.org/category/ guias-clinicas/antirretroviral-vigentes/ (acceso el 31 de julio de 2020).

3.- DHHS Panel on Antiretroviral Guidelines for Adults and Adolescents. Guidelines for the use of antiretroviral agents in adults and adolescents living with HIV. December 2019. https://aidsinfo.nih.gov/guidelines/html/1/adultand-adolescent-arv/0 (acceso el 31 de julio de 2020)

4.- Laprise C, Pokomandy A, Baril J G, Dufresne $\mathrm{S}$, Trottier $\mathrm{H}$. virologic failure following persistent low-level viremia in a cohort of
HIV-positive patients: results from 12 years of observation. Clin Infect Dis 2013; 57 (10): 1489-96. https://doi.org/10.1093/cid/cit529.

5.- Helou E, Shenoi, S, Kyriakides T, Landry M L, Kozal M, Barakat L A. Characterizing patients with very-low-level hiv viremia: a community-based study. J Int Assoc Provid AIDS Care 2017; 16 (3): 261-6. https://doi. org/10.1177/2325957416680028.

6.- Teira R, Vidal F, Muñoz Sánchez P, Geijo P, Viciana P, Ribera P, et al. Very low level viraemia and risk of virological failure in treated HIV-1 infected patients. HIV Med 2017; 18 (3):196-203. https://doi.org/10.1111/ hiv. 12413.

7.- Hermans L E, Moorhpuse M, Carmona M, Grobbee D E, Hofstra L M, Richman D D, et al. Effect of HIV-1 low level viraemia during antiretroviral therapy on treatment outcomes in WHO- guided South African treatment programmes: a multicentre cohort study. Lancet Infect Dis 2018; 18 (2): 188-97. https://doi. org/10.1016/s1473-3099(17)30681-3.

8.- Joya C, Hyun Won S, Schofield C, Lalani T,
Maves R C, Kronmann K, et al. Persistent low level viremia while on antiretroviral therapy is an independent risk factor for virological failure. Clin Infect Dis 2019; 69 (12): 2145-52. https://doi.org/10.1093/cid/ciz129.

9.- Vandenhende M A, Ingle S, May M, Chene G, Zangerle R, Van Sighem A, et al. Impact of low level viremia on clinical and virological outcomes in treated HIV-1 infected patients. AIDS 2015; 29 (3): 373-83. https://doi. org/10.1097/QAD.0000000000000544.

10.- Fleming J, Mathews C, Rutstein R, Aberg J, Somboonwit C, Cheever L, et al. Low-level viremia and virologic failure in persons with HIV infection treated with antiretroviral therapy. AIDS 2019, 33: 2005-2012. https:// doi:10.1097/QAD.0000000000002306.

11.- Pernas B, Grandal M, Pertega S, Cañizares A, Castro-Iglesias A, Mena A, et al. Any impact of blips and low-level viraemia episodes among HIIV infected patients with sustained virological suppression on ART?. J Antimicrobial Chemother 2016; 71: 1051-5. https://doi.org/10.1093/jac/dkv433. 